\title{
Differential Signaling and Virus Production in Calu-3 Cells and Vero Cells upon SARS-CoV-2 Infection
}

\author{
Byoung Kwon Park ${ }^{1, \dagger}$, Dongbum Kim ${ }^{1, \dagger}$, Sangkyu Park ${ }^{2, \dagger}$, Sony Maharjan ${ }^{1, \dagger}$, Jinsoo Kim ${ }^{3, \dagger}$, Jun-Kyu Choi ${ }^{2}$, \\ Madhav Akauliya ${ }^{3}$, Younghee Lee ${ }^{2, *}$ and Hyung-Joo Kwon ${ }^{1,3, *}$ \\ ${ }^{1}$ Institute of Medical Science, College of Medicine, Hallym University, Chuncheon 24252, \\ ${ }^{2}$ Department of Biochemistry, College of Natural Sciences, Chungbuk National University, Cheongju 28644, \\ ${ }^{3}$ Department of Microbiology, College of Medicine, Hallym University, Chuncheon 24252, Republic of Korea
}

\begin{abstract}
Severe acute respiratory syndrome CoV-2 (SARS-CoV-2) is responsible for the current coronavirus disease 2019 (COVID-19) pandemic. Signaling pathways that are essential for virus production have potential as therapeutic targets against COVID-19. In this study, we investigated cellular responses in two cell lines, Vero and Calu-3, upon SARS-CoV-2 infection and evaluated the effects of pathway-specific inhibitors on virus production. SARS-CoV-2 infection induced dephosphorylation of STAT1 and STAT3, high virus production, and apoptosis in Vero cells. However, in Calu-3 cells, SARS-CoV-2 infection induced long-lasting phosphorylation of STAT1 and STAT3, low virus production, and no prominent apoptosis. Inhibitors that target STAT3 phosphorylation and dimerization reduced SARS-CoV-2 production in Calu-3 cells, but not in Vero cells. These results suggest a necessity to evaluate cellular consequences upon SARS-CoV-2 infection using various model cell lines to find out more appropriate cells recapitulating relevant responses to SARS-CoV-2 infection in vitro.
\end{abstract}

Key Words: Apoptosis, COVID-19, SARS-CoV-2, STAT1, STAT3, STAT3 phosphorylation

\section{INTRODUCTION}

Coronaviruses (CoVs), single-stranded RNA viruses with a crown-shaped morphology, cause diseases in humans and animals. Human coronaviruses (HCoVs) include endemic alpha-coronaviruses (HCoV-229E and HCoV-NL63) and betacoronaviruses (HCoV-OC43 and HCoV-HKU1) that infect the upper respiratory system and cause the common cold (Fehr and Perlman, 2015). From 2002-December 2003, Severe Acute Respiratory Syndrome CoV (SARS-CoV) emerged as a fatal zoonotic pathogen that caused 8,096 infections and 774 deaths globally, according to a report by the World Health Organization (WHO) (WHO, 2015). SARS-CoV infects the lower respiratory tract in humans and causes bronchitis and pneumonia symptoms (Holmes, 2003; Peiris et al., 2004; Girard et al., 2005). SARS-CoV belongs to lineage B of betacoronaviruses. In 2012, another CoV, belonging to lineage C of beta-coronaviruses, was isolated in Saudi Arabia and named Middle East Respiratory Syndrome-related coronavi- rus (MERS-CoV; Zaki et al., 2012). The WHO reported that 2,494 people in 27 countries have been infected with MERSCoV since September 2012 and that 858 people have died (fatality rate of $34 \%$ ) (WHO, 2019). The symptoms of MERSCoV and SARS-CoV infections are similar and include fever, upper and lower respiratory tract infections, cough, vomiting, diarrhea, liver cirrhosis, pneumonia, and multiple organ failure (Zhang et al., 2016). Recently, Severe Acute Respiratory Syndrome CoV-2 (SARS-CoV-2), which belongs to lineage B of beta-coronaviruses, first appeared in Wuhan City, China in December 2019 and is the cause of the current coronavirus disease 2019 (COVID-19) pandemic (Khalaf et al., 2020). On December 06, 2020, the WHO reported that $65,872,391$ people in numerous countries have been infected with SARSCoV-2 since January 2020, and 1,523,656 people have died (estimated global mortality of $2.31 \%$ ) (WHO, 2020).

COVID-19 patients have common symptoms, such as fever, cough, and fatigue, along with sputum production and lymphopenia. Severe pneumonia has been diagnosed in COVID-19

\section{Open Access https://doi.org/10.4062/biomolther.2020.226}

This is an Open Access article distributed under the terms of the Creative Commons Attribution Non-Commercial License (http://creativecommons.org/licenses/by-nc/4.0/) which permits unrestricted non-commercial use, distribution, and reproduction in any medium, provided the original work is properly cited.
Received Dec 14, 2020 Revised Dec 30, 2020 Accepted Jan 4, 2021 Published Online Jan 28, 2021

\section{*Corresponding Authors}

E-mail: hjookwon@hallym.ac.kr (Kwon HJ), yhl4177@cbnu.ac.kr (Lee Y) Tel: +82-33-248-2635 (Kwon HJ), +82-43-261-3387 (Lee Y) Fax: +82-33-241-3640 (Kwon HJ), +82-43-267-2306 (Lee Y) ${ }^{\dagger}$ The first five authors contributed equally to this work. 
patients based on clinical features of chest CT scans. Additionally, abnormal features, such as RNAemia, acute respiratory distress syndrome (ARDS), acute cardiac injury, incidence of grand-glass opacities, and significantly high levels of serum cytokines and chemokines, have also been observed in COVID-19 patients (Rothan and Byrareddy, 2020). Investigation of immune responses in COVID-19 patients will guide proper COVID-19 treatment (McKechnie and Blish, 2020). Due to high expression of interferons and interferon-stimulated genes (ISGs) in patients with severe cases of COVID-19, finely tuned interferon therapy should be considered (Park and Iwasaki, 2020). SARS-CoV, MERS-CoV, and SARS-CoV-2 infections have led to severe health hazards involving high global spread and high mortality. However, effective therapeutics and vaccines against these viral infections are currently unavailable.

SARS-CoV-2 was isolated from human airway epithelial cells and amplified in Vero E6 and Huh7 cell lines (Datta et al., 2020; Matsuyama et al., 2020). SARS-CoV-2 expresses several proteins, including the spike (S), membrane (M), envelope $(\mathrm{E})$, nucleocapsid $(\mathrm{N})$, and accessory proteins (Datta et al., 2020; Xia et al., 2020). The S proteins on the viral membranes of SARS-CoV-2 and SARS-CoV interact with angiotensin-converting enzyme 2 (ACE2) on the membranes of host cells and then, depending on transmembrane protease serine 2 (TMPRSS2) activity, the virus enters the cell (Matsuyama et al., 2020). It has also been shown that cathepsin B/L can substitute for TMPRSS2 activity (Datta et al., 2020; Matsuyama et al., 2020). Thus, several studies have investigated the ACE2 receptor, TMPRSS2, and cathepsin B/L as potential therapeutic targets for the treatment of SARS-CoV-2 infection (Datta et al., 2020; Xia et al., 2020).

STAT3 is a two-faced antiviral or proviral regulator, depending on the virus type and the host cell type (Kuchipudi, 2015; Chang et al., 2018). Phosphorylation and activation of STAT3 is involved in replication and pathogenesis of various viruses, including Epstein-Barr virus (EBV), human immunodeficiency virus type 1 (HIV-1), hepatitis B virus (HBV), and varicella-zoster virus (VZV; Waris et al., 2001; Sen et al., 2012; Del Cornò et al., 2014), and several viral proteins are known to activate STAT3 by enhancing its phosphorylation (Roca Suarez et al., 2018). On the other hand, downregulation of STAT3 activity via dephosphorylation and degradation of STAT3 occurs during influenza A virus (IAV), hepatitis E virus (HEV), and SARSCoV infections, suggesting that STAT3 is an important mediator of host immune responses to viruses (Mizutani et al., 2004; Chandra et al., 2008; Hui et al., 2016). The role of STAT3 in response to infection by the same virus may differ based on the specific host cell type infected.

STAT1 and STAT2 are involved in interferon (IFN) signaling, which is stimulated by viral infections (Nan et al., 2017). When IFN signaling is activated, STAT1 is phosphorylated and forms homodimers or heterodimers with STAT2 or STAT3. However, the function of STAT3 is controversial. Although phosphorylated STAT3 is known to bind and activate the promoters of interferon-stimulated genes (ISGs; Heim, 2015), indicating antiviral activity, negative regulation of antiviral responses by STAT3 has also been described for the later phase of viral infection, possibly by quenching STAT1 activity via STAT1/ STAT3 dimerization (Ho and Ivashkiv, 2006; Wang et al., 2011). Although the exact functions of STAT1 and STAT3 in response to SARS-CoV-2 infection have not yet been investigated, STAT1 and STAT3 may have potential as therapeutic targets for the treatment of SARS-CoV-2 infection (Roca Suarez et al., 2018).

In this study, we investigated the cellular responses and virus production in Vero cells and Calu- 3 cells after infection with SARS-CoV-2. We found different patterns of STAT1 and STAT3 phosphorylation in the two cell lines after virus infection. We also investigated the effects of inhibitors that target STAT3 phosphorylation (JAK inhibitor I) and dimerization (STA-21, S3I201) on SARS-CoV-2 production in Calu-3 cells.

\section{MATERIALS AND METHODS}

\section{Cell culture and viruses}

African green monkey kidney Vero and Vero E6 cells and human airway epithelial Calu-3 cells were obtained from the Korean Cell Line Bank (Seoul, Korea). The cells were cultured in Dulbecco's modified Eagle's medium (DMEM, Thermo Fisher Scientific, Waltham, MA, USA) containing $10 \%$ fetal bovine serum (FBS, Thermo Fisher Scientific), 25 mM HEPES, $100 \mathrm{U} / \mathrm{mL}$ penicillin, and $100 \mu \mathrm{g} / \mathrm{mL}$ streptomycin. The cells were incubated in $95 \%$ air and $5 \% \mathrm{CO}_{2}$ at $37^{\circ} \mathrm{C}$. SARS-CoV-2 (NCCP No. 43326) was obtained from the National Culture Collection for Pathogens (Osong, Korea).

\section{Virus amplification}

Vero cells $\left(2 \times 10^{5}\right.$ cells/well in 6-well plates) were cultured in DMEM containing $10 \% \mathrm{FBS}$ at $37^{\circ} \mathrm{C}$ in a $\mathrm{CO}_{2}$ incubator overnight (Park et al., 2019; Kandeel et al., 2020). The cells were washed with phosphate-buffered saline (PBS), SARS-CoV-2 in PBS at $\mathrm{MOI} 0.01$ was added into each well, and the plates were incubated for $1 \mathrm{~h}$ at $37^{\circ} \mathrm{C}$ in a $\mathrm{CO}_{2}$ incubator. After incubation, $2 \mathrm{~mL}$ of DMEM containing $2 \%$ FBS was added to each well and the plates were incubated at $37^{\circ} \mathrm{C}$ in a $\mathrm{CO}_{2}$ incubator for an additional 3 days. Cell culture supernatants were harvested and centrifuged at 2,000 rpm for $10 \mathrm{~min}$ at $4^{\circ} \mathrm{C}$ to remove cell debris. The amount of virus in the supernatants was quantified by plaque assay and $400-\mu \mathrm{L}$ aliquots $\left(5 \times 10^{6}\right.$ $\mathrm{pfu} / \mathrm{mL}$ ) of virus were stored at $-70^{\circ} \mathrm{C}$. SARS-CoV-2 amplification and cell culture procedures were performed according to biosafety level 3 (BSL-3) conditions.

\section{Virus quantification by plaque formation assay}

Vero E6 cells $\left(7 \times 10^{5}\right.$ cells/well) were cultured in 6 -well plates (Corning, NY, USA) for $12 \mathrm{~h}$. The cells were washed with PBS and infected with 10-fold serial dilutions of SARSCoV-2. After $1 \mathrm{~h}$ of incubation, supernatants were removed, and the wells were replenished with $3 \mathrm{~mL}$ DMEM/F12 medium (Thermo Fisher Scientific) containing $2 \%$ Oxoid agar and $N-p$ Tosyl-L-phenylalanine chloromethyl ketone (TPCK, $1 \mu \mathrm{g} / \mathrm{mL}$ )treated trypsin. Plaques were allowed to develop for $72 \mathrm{~h}$ at $37^{\circ} \mathrm{C}$. Plates were stained with $0.1 \%$ crystal violet for $1 \mathrm{~h}$ to evaluate plaque formation.

\section{Reagents and virus infection}

Antibodies to STAT1 (Catalog No. 14994S), phosphoSTAT1 (Tyr-701, Catalog No. 9167S), STAT3 (Catalog No. 12640S), phospho-STAT3 (Tyr-705, Catalog No. 9145S), polyADP ribose polymerase (PARP, Catalog No. 9542S), cleaved Caspase-3 (Catalog No. 9661S), c-Myc (Catalog No. 5605S), and cyclin D1 (Catalog No. 2978S) were purchased from Cell Signaling Technology (Beverly, MA, USA). Anti- $\beta$-actin mono- 
clonal antibody was obtained from Sigma-Aldrich (Saint Louis, MO, USA).

MAPK/ERK kinase (MEK) inhibitor PD 98059 (Catalog No. P215), p38 inhibitor PD 169316 (Catalog No. P9248), stressactivated protein kinase (SAPK)/c-Jun $\mathrm{N}$-terminal kinase (JNK) inhibitor SP 600125 (Catalog No. S5567), JAK2 kinase inhibitor AG490 (Catalog No. T3434), STAT3 dimerization inhibitor S3I-201 (Catalog No. SML0330), and STAT3 dimerization inhibitor STA-21 (Catalog No. SML2161) were purchased from Sigma-Aldrich. JAK inhibitor I (Catalog No. 420099), a potent ATP-competitive inhibitor of JAK1, JAK2, and JAK3, was purchased from Calbiochem (San Diego, CA, USA). All the inhibitors were dissolved in dimethyl sulfoxide (DMSO, Catalog No. 10378-73, Kanto Chemical, Tokyo, Japan). In the inhibitor assays, cells were preincubated with each inhibitor for 30 min before infection with SARS-CoV-2 at 0.1 or $0.5 \mathrm{MOI}$ for $1 \mathrm{~h}$ and then the cells were cultured in DMEM containing $2 \%$ FBS for $48 \mathrm{~h}$ or $72 \mathrm{~h}$.

\section{Western blot analysis}

Cell lysates from mock-infected Vero and Calu- 3 cells and SARS-CoV-2-infected cells were prepared with cell lysis buffer (20 mM Tris-HCl pH 8.0, 5 mM EDTA, 150 mM NaCl, 100 mM $\mathrm{NaF}, 2 \mathrm{mM}$ Na3VO4, 1\% NP-40) and centrifuged at 14,000 rpm at $4{ }^{\circ} \mathrm{C}$ for $20 \mathrm{~min}$. Equal amounts of protein were separated on $4-12 \%$ Bis-Tris gradient gels (Thermo Fisher Scientific) and transferred onto nitrocellulose membranes. The membranes were blocked and incubated with antibody overnight at $4^{\circ} \mathrm{C}$. Then, the membranes were incubated with a horseradish peroxidase-conjugated secondary antibody and immunoreactive bands were detected using an enhanced chemiluminescence $(E C L)$ reagent (Thermo Fisher Scientific).

\section{Confocal images}

Induction of STAT3 phosphorylation and phospho-STAT3 nuclear localization by SARS-CoV-2 were observed by indirect immunofluorescence and confocal microscopy. Calu-3 cells were cultured on glass coverslips in 12-well plates overnight. The cells were pretreated with DMSO, $25 \mu \mathrm{M}$ AG490, 1 $\mu \mathrm{M}$ JAK inhibitor I, $20 \mu \mathrm{M}$ S3I-201, or $10 \mu \mathrm{M}$ STA-21 for 30 min and then infected with SARS-CoV-2 in PBS (0.5 MOI) for $1 \mathrm{~h}$ at $37^{\circ} \mathrm{C}$. The cells were cultured in DMEM containing $2 \%$ FBS for $48 \mathrm{~h}$ and then treated with Leptomycin B (LMB, Cell Signaling Technology, $20 \mathrm{nM}$ ), an inhibitor of the nuclear export receptor CRM1, for $3 \mathrm{~h}$. The cells were fixed with $4 \%$ paraformaldehyde in PBS, permeabilized with $0.1 \%$ Triton $X-100$, and then blocked with $3 \%$ BSA. To detect STAT3 phosphorylation and phospho-STAT3 nuclear localization, cells were stained with phospho-STAT3 antibody (Cell Signaling Technology) for $2 \mathrm{~h}$ at room temperature. The cells were washed with PBST (0.1\% Triton $\mathrm{X}-100$ in PBS) containing $1 \% \mathrm{BSA}$ and stained with Alexa Flour 488-conjugated secondary antibody (Thermo Fisher Scientific) for $1 \mathrm{~h}$. Nuclei were stained with Hoechst 33258 (Thermo Fisher Scientific). The samples were observed by confocal laser scanning microscopy (CLSM, LSM 710, Carl Zeiss, Jena, Germany).

\section{Investigation of SARS-CoV-2 production in the presence of pathway-specific inhibitors}

Vero cells $\left(5 \times 10^{4}\right.$ cells/well in 12 -well plates) and Calu-3 cells $\left(5 \times 10^{4}\right.$ cells/well in 12 -well plates $)$ were cultured overnight. To investigate the effect of pathway-specific kinase in- hibitors and STAT3 dimerization inhibitors (S3I-201 and STA21) on SARS-CoV-2 production, cells were pretreated with DMSO, $1 \mu \mathrm{M}$ JAK inhibitor I, $20 \mu \mathrm{M}$ S3I-201, or $10 \mu \mathrm{M}$ STA-21 for 30 min prior to virus infection. After cells were infected with SARS-CoV-2 in PBS (0.1 MOI) for $1 \mathrm{~h}$ at $37^{\circ} \mathrm{C}, 2 \mathrm{~mL}$ of DMEM containing $2 \%$ FBS was added to each well and the plates were incubated for an additional $72 \mathrm{~h}$. Supernatants of virusinfected cells were collected and virus replication was quantified using real-time RT-PCR and the plaque formation assay.

\section{Quantitative real-time RT-PCR (qRT-PCR)}

Viral particles were collected from virus-infected cell culture supernatants $(100 \mu \mathrm{L})$ and viral RNAs were isolated from the supernatants using the QIAamp Viral RNA Mini Kit (Catalog No. 52904, Qiagen, Hilden, Germany) according to the manufacturer's instructions. cDNA $(50 \mu \mathrm{L})$ was synthesized using the Reverse Transcription System Kit (Catalog No. A3500, Promega, Madison, WI, USA). To quantify expression of the RNA-dependent RNA polymerase $(R d R P)$ gene of SARSCoV-2, the following primers were used (Kim et al., 2020): forward primer, 5'-GTGAAATGGTCATGTGTGGCGG-3', reverse primer 5'-CAAATGTTAAAAACACTATTAGCATA-3', and TaqMan Probe 5'-FAM-CAGGTGGAACCTCATCAGGAGATGCTAMRA-3'. Primers and the probe were synthesized by Genotech (Daejeon, Korea). $10 \mu \mathrm{L}$ of GoTaq Probe qPCR Master Mix (Catalog No. A6101, Promega) was added to $10 \mu \mathrm{L}$ of the reaction mixture containing $125 \mathrm{nM}$ each of the forward and reverse primers, $250 \mathrm{nM}$ probe, and $1 \mu \mathrm{L}$ of cDNA. After denaturation at $95^{\circ} \mathrm{C}$ for $5 \mathrm{~min}, 45$ cycles at $95^{\circ} \mathrm{C}$ for 15 sec and $60^{\circ} \mathrm{C}$ for 1 min were performed in the Rotor-Gene $Q$ real-time PCR cycler (Qiagen). The numbers of copies of the $R d R P$ gene in the samples were calculated using an $R d R P$ cDNA standard curve.

\section{Statistical analysis}

Results are shown as the mean \pm standard deviation. Differences between the samples were analyzed using an unpaired, 2-tailed nonparametric $t$-test of significance (Instat; GraphPad Inc., San Diego, CA, USA). $p$-values $<0.05$ were considered statistically significant.

\section{RESULTS}

\section{Differential virus production and apoptosis of Vero and Calu-3 cells in response to SARS-CoV-2 infection}

The cellular response to virus infection can differ depending on the infected cell type, therefore we used two different host cell lines expressing SARS-CoV-2 receptor ACE2, Vero and Calu-3 (Shang et al., 2020). Vero cells originate from African green monkey kidney and Calu-3 cells originate from human airway epithelium. The results of qRT-PCR showed that the amount of viral RNA was higher in Vero cells than in Calu-3 cells after SARS-CoV-2 infection (Fig. 1A). Virus production was also confirmed by plaque formation assay. As shown in Fig. 1B, virus production was higher in Vero cells than in Calu3 cells after SARS-CoV-2 infection.

Higher production of virus can be associated with higher apoptosis of host cells. Therefore, we investigated expression of proliferation and apoptosis markers. We infected Vero cells and Calu- 3 cells with SARS-CoV- 2 and measured the levels of cleaved PARP and cleaved Caspase- 3 proteins up 

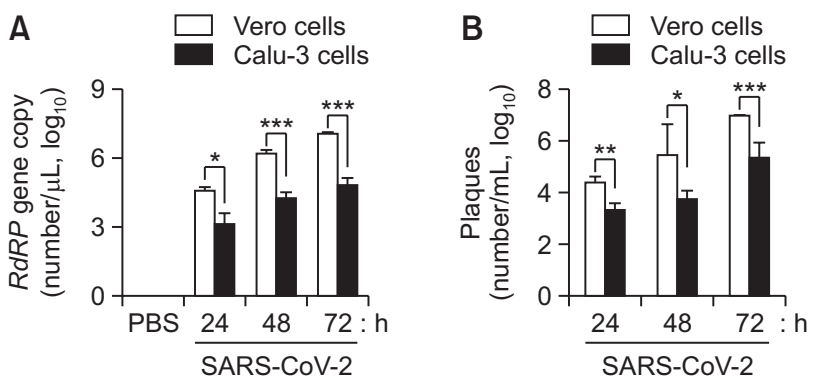

Fig. 1. Differential production of SARS-CoV-2 in Vero and Calu3 cells. Vero and Calu- 3 cells $\left(5 \times 10^{4}\right.$ cells/well in 12-well plates) were cultured in DMEM containing 10\% FBS. After overnight culture, cells were washed with PBS and SARS-CoV-2 in PBS at a $\mathrm{MOI}$ of 0.1 was added into each well $(n=3)$. After $1 \mathrm{~h}$ of incubation, the medium was replenished with DMEM containing $2 \%$ FBS. (A) Supernatants of virus-infected cell cultures were collected at the indicated times after virus infection to isolate viral RNAs. Virus replication was quantified by real-time RT-PCR analysis of the SARSCoV-2 RdRP gene. Copy numbers of the RdRP gene in $1 \mu \mathrm{L}$ of the cDNA samples were calculated using a standard curve of RdRP cDNA. (B) Supernatants of virus-infected Vero and Calu-3 cells (12-well plates) were collected at the indicated times after virus infection. Virus production was quantified by plaque formation assay. ${ }^{*} p<0.05,{ }^{* *} p<0.01,{ }^{* * *} p<0.001$.

to $72 \mathrm{~h}$ post infection. In Vero cells infected with SARS-CoV-2, the levels of cleaved PARP and cleaved Caspase- 3 proteins increased significantly at $72 \mathrm{~h}$ (Fig. 2A). Compared with the mock-infected control, expression of c-Myc and cyclin D1, which are required for cell proliferation, decreased in Vero cells upon virus infection (Fig. 2A). The Vero E6 cell line is a derivative of the Vero cell line. Like Vero cells, the levels of cleaved PARP and cleaved Caspase-3 proteins increased and c-Myc and cyclinD1 proteins were decreased in SARSCoV-2-infected Vero E6 cells during the culture period (Supplementary Fig. 1). In Calu-3 cells infected with SARS-CoV-2, the level of cleaved PARP protein increased only slightly and no cleaved Caspase-3 protein was present (Fig. 2B). Furthermore, c-Myc and cyclin D1 expression was maintained in SARS-CoV-2-infected Calu-3 cells and mock-infected Calu-3 cells (Fig. 2B). Therefore, apoptosis occurred in Vero cells, but not in Calu-3 cells, upon SARS-CoV-2 infection.

\section{Effect of SARS-CoV-2 infection on STAT1 and STAT3 phosphorylation in Vero and Calu-3 cells}

Because STAT1 and STAT3 are associated with cellular apoptosis and also known to contribute to virus production and pathogenesis, we determined phosphorylation levels of STAT1 (Tyr-701) and STAT3 (Tyr-705) in Vero and Calu-3 cells in response to SARS-CoV-2 infection. During the culture period, phosphorylation of STAT1 and STAT3 increased in mock-infected Vero cells. When we infect the cells with SARS-COV-2 or perform mock infection, we wash the cells with PBS, mock-infect or infect the viruses in PBS for $1 \mathrm{~h}$. Then, the cells were cultured in a fresh DMEM medium containing $2 \%$ FBS. Therefore, this procedure can be a kind of factor-starvation for cells. We suppose that some unidentified changes resulting in STAT phosphorylation autonomously occurs in the mock-infected Vero cells. However, phosphorylation of STAT1 and STAT3 decreased drastically at $48 \mathrm{~h}$ in Vero cells infected with SARS-CoV-2 (Fig. 3A). These results are similar with the previous data published by other investigators regarding SARS-CoV infection in Vero E6 cells (Mizutani et al., 2004). In mock-infected Calu-3 cells, there was only a basal level of STAT phosphorylation. In Calu-3 cells infected with SARS-CoV-2, there was a gradual increase in phosphorylation of STAT1 and STAT3 up to $48 \mathrm{~h}$ and then a slight decrease at $72 \mathrm{~h}$ (Fig. 3B). A multiplicity of infection (MOI)-dependent increase of STAT1 and STAT3 phosphorylation after infection was detected up to $0.5 \mathrm{MOI}$ of SARS-CoV-2. STAT1 and STAT3 phosphorylation was similar in Vero cells and Vero E6 cells. During the culture period, phosphorylation of STAT1 and STAT3 increased in mock-infected Vero E6 cells. When compared with mock-infected Vero E6 cells, STAT1 and STAT3 phosphorylation decreased at $48 \mathrm{~h}$ in SARS-CoV-2-infected Vero E6 cells, suggesting dephosphorylation by SARS-CoV-2 infection (Supplementary Fig. 2).

\section{Effects of pathway-specific inhibitors on SARS-CoV-2- induced STAT1 and STAT3 phosphorylation}

Because STAT family members are phosphorylated by several kinases, including Janus kinases (JAKs) and mitogenactivated protein kinases (MAPKs) (Rawlings et al., 2004), we examined the intracellular signals in response to SARSCoV-2 infection in Calu-3 cells. We used pathway-specific kinase inhibitors to determine whether SARS-CoV-2 modulates phosphorylation of STAT1 and STAT3 via the MAPK or JAK pathway. STAT1 phosphorylation by SARS-CoV-2 infection was inhibited by the p38 MAPK inhibitor PD 169316, but not by the MAPK/ERK kinase (MEK) inhibitor PD 98059 or the stress-activated protein kinase (SAPK)/c-Jun N-terminal kinase (JNK) inhibitor SP 600125. STAT1 phosphorylation was also reduced by JAK inhibitor I, a potent ATP-competitive inhibitor of JAK1, 2, and 3. However, the JAK2 inhibitor AG490 did not affect STAT1 phosphorylation, suggesting that JAK1 and JAK3, but not JAK2, are involved in STAT1 phosphorylation. STAT3 phosphorylation was also inhibited by JAK inhibitor I (Fig. 4A).

Previously, small-molecule inhibitors that target STAT3 dimerization (e.g., STA-21, S3I-201) were evaluated as antivirals in vitro and in animal models (Roca Suarez et al., 2018). We investigated the effect of S3I-201 on STAT1 and STAT3 phosphorylation. S3I-201 $(1-100 \mu \mathrm{M})$ did not affect STAT1 and STAT3 phosphorylation induced by SARS-CoV-2 infection, and this result supports the idea that STAT3 dimerization occurs downstream of STAT3 phosphorylation (Fig. 4B). We tested the inhibitors on mock-infected cells and found that they had minimal effects, with the exception of JAK inhibitor I that reduced basal levels of STAT1 and STAT3 phosphorylation (Fig. 4C). We used immunostaining and confocal microscopy to confirm phosphorylation of STAT3 in response to SARS-CoV-2 infection and inhibition of STAT3 phosphorylation by JAK inhibitor I. When Leptomycin B was used to block nuclear export, phosphorylation and nuclear localization of STAT3 was abundant, suggesting that phosphorylated STAT3 is translocated into the nucleus after SARS-CoV-2 infection. In contrast, phosphorylation and nuclear localization of STAT3 was clearly reduced in the presence of JAK inhibitor I. S3I-201 did not affect STAT1 and STAT3 phosphorylation but reduced nuclear localization of STAT3 probably because of dimerization inhibition (Fig. 4D). 
A Vero cells

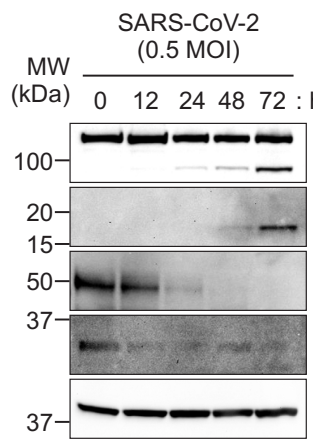

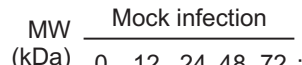
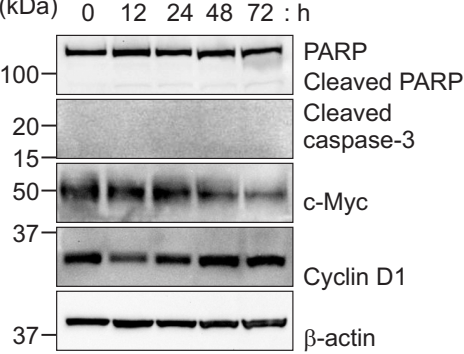

B Calu-3 cells

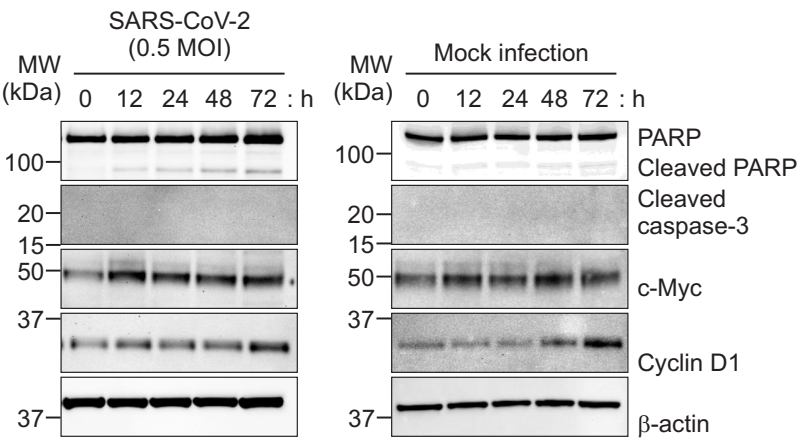

Fig. 2. Effect of SARS-CoV-2 on induction of apoptosis. (A, B) Vero (A) and Calu-3 (B) cells were infected with $0.5 \mathrm{MOI}$ of SARS-CoV-2 or without virus for the indicated times. After $1 \mathrm{~h}$ of incubation, the medium was replenished with DMEM containing $2 \%$ FBS. After $72 \mathrm{~h}$ of incubation, cell lysates were prepared, and Western blot analysis was performed to detect PARP, cleaved Caspase-3, c-Myc, and cyclin D1. $\beta$-actin was used as the control.

A Vero cells

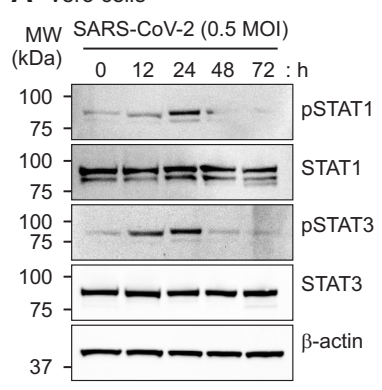

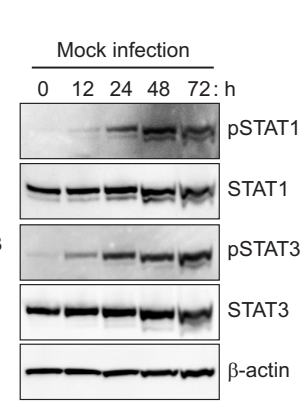
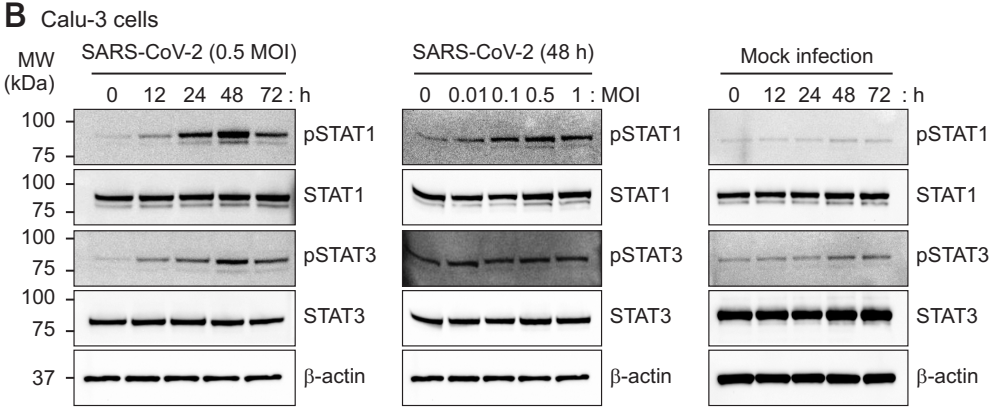

Fig. 3. STAT1 and STAT3 phosphorylation in SARS-CoV-2-infected Vero and Calu-3 cells. (A) Vero cells were infected with $0.5 \mathrm{MOI}$ of SARS-CoV-2 (left) or were mock-infected (right) for the indicated times. (B) Calu- 3 cells were infected with $0.5 \mathrm{MOI}$ of SARS-CoV-2 (left), indicated MOls (middle), or were mock-infected cells (right) for the indicated times with the indicated MOls. Western blotting of the cell lysates was performed with the indicated antibodies. $\beta$-actin was used as the control.

\section{Effects of pathway-specific inhibitors on SARS-CoV-2 production}

To evaluate the effects of inhibitors that target STAT3 activation and function on SARS-CoV-2 production, we pretreated Vero and Calu-3 cells with DMSO, JAK inhibitor I, S3I-201, or STA-21 and then measured SARS-CoV-2 production using a plaque assay and qRT-PCR. In Vero cells, none of the inhibitors affected SARS-CoV-2 production. However, treatment with JAK inhibitor I, S3I-201, or STA-21 reduced SARS-CoV-2 production 30-100-fold in Calu-3 cells (Fig. 5). Therefore, the inhibition of STAT3 phosphorylation by JAK inhibitor I and the inhibition of pSTAT3 dimerization by S3I-201 or STA-21 hampered SARS-CoV-2 production suggesting that STAT3 phosphorylation and nuclear translocation is required. Cytotoxicity of the compounds at the concentrations used was minimal in Calu-3 cells (Supplementary Fig. 3).

\section{DISCUSSION}

STAT3 is known to regulate virus infection positively or negatively. The biology of STAT3 activation and regulation in response to SARS-CoV-2 infection has not yet been investigated in detail. By understanding the role of STAT3 in CoV pathophysiology, targeted therapeutics against STAT3 may be effective against CoVs, including the current pandemic SARSCoV-2. In this study, we found that STAT3 is activated in Calu3 cells upon SARS-CoV-2 infection and that STAT3-targeted inhibitors suppressed SARS-CoV-2 production.

SARS-CoV and SARS-CoV-2 enter host cells via ACE2 (Datta et al., 2020). The kinetics of STAT phosphorylation after virus infection likely depends on the host cell type. Our results showed that SARS-CoV-2 stimulated phosphorylation of STAT1 and STAT3 in Calu-3 cells. In contrast, STAT1 and STAT3 phosphorylation occurred spontaneously in Vero and Vero E6 cells during the culture period without virus infection. In the SARS-CoV-2-infected Vero and Vero E6 cells, the phosphorylation levels of STAT1 and STAT3 decreased at $48 \mathrm{~h}$ after infection. Therefore, it appears that SARS-CoV-2 infection induces dephosphorylation of STAT1 and STAT3 in Vero and Vero E6 cells, consistent with what was reported for SARSCoV infection (Mizutani et al., 2004). Regulation of apoptosis after virus infection also differed based on the cell type. Three days after SARS-CoV-2 infection, expression of pro-apoptotic proteins increased drastically in Vero cells and Vero E6 cells, but not in Calu-3 cells. In contrast, c-Myc and Cyclin D1 expression diminished in Vero cells, but was maintained in Calu3 cells. These results suggest that prominent apoptosis occurs in Vero cells, but not in Calu-3 cells, after virus infection. 
A

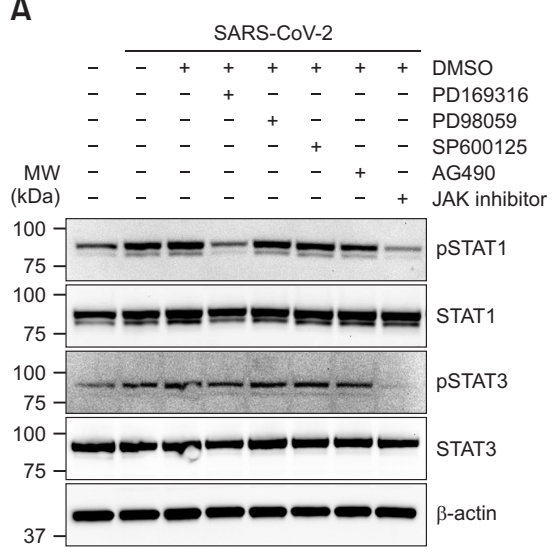

B

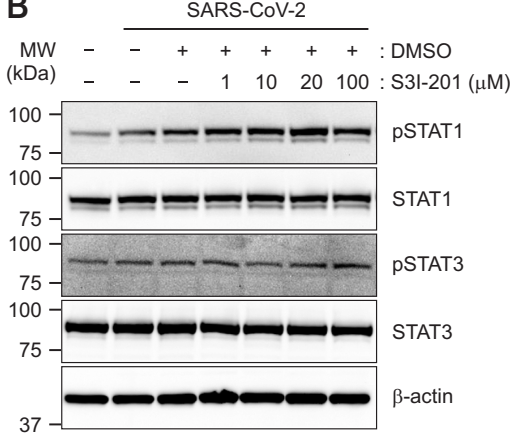

C



MW - $-\quad-\quad-+-$ JAK inhibitor

$(\mathrm{kDa})-\quad-\quad-\quad-\quad+\mathrm{S} 3 \mathrm{l}-201$

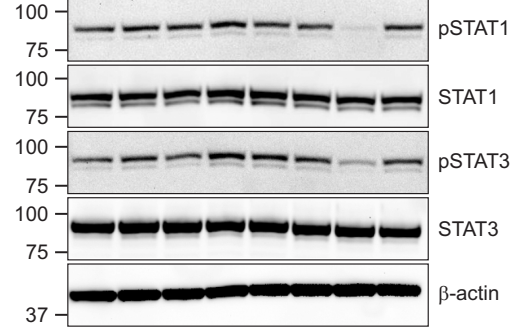

D

Anti-pSTAT3 Ab

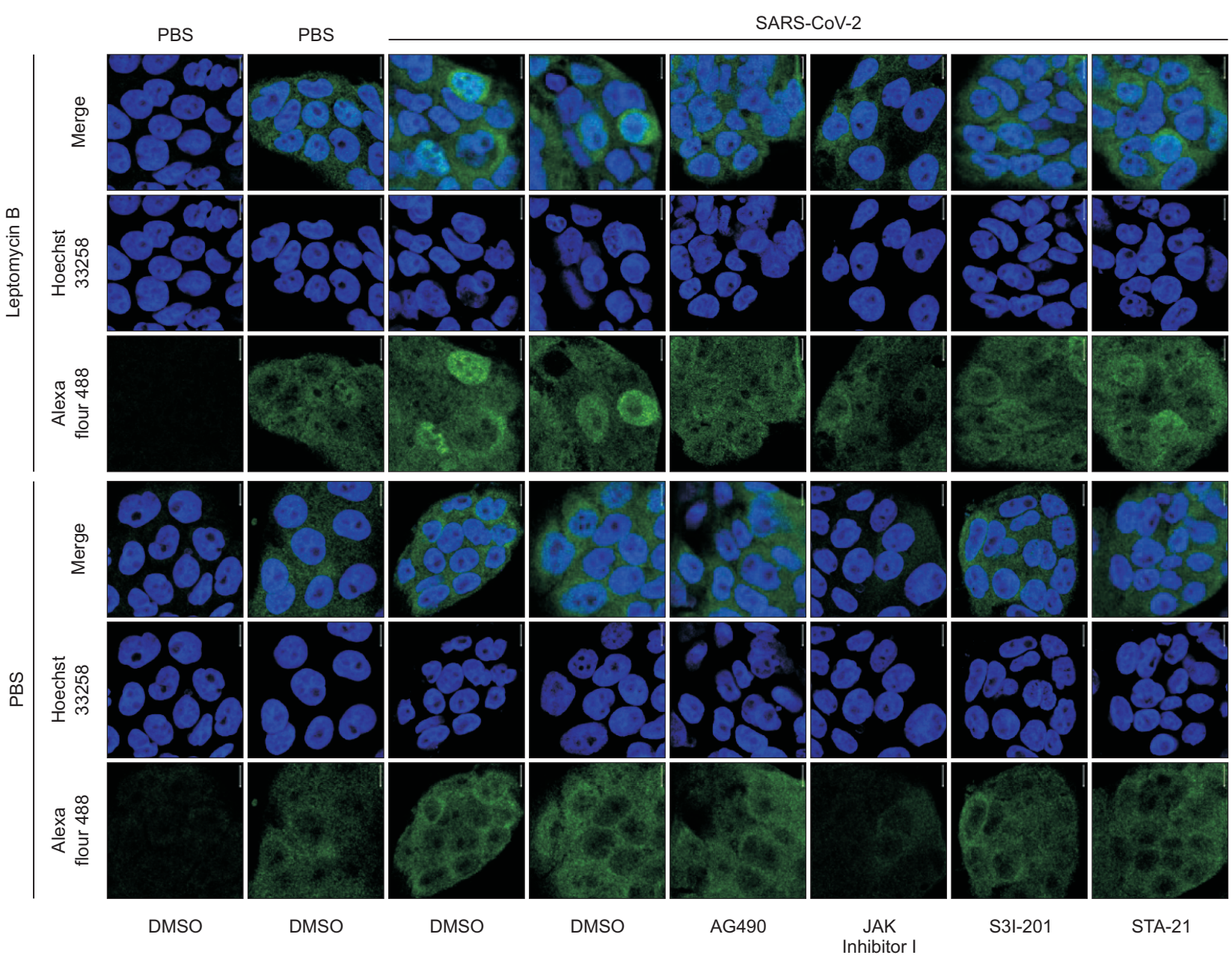

Fig. 4. Effects of pathway-specific inhibitors on SARS-CoV-2-induced STAT1 and STAT3 phosphorylation in Calu-3 cells. (A) Calu-3 cells were pretreated with 0.1\% DMSO, $10 \mu \mathrm{M}$ PD169316, $25 \mu \mathrm{M}$ PD98059, $25 \mu \mathrm{M}$ SP600125, $25 \mu \mathrm{M}$ AG490, or $1 \mu \mathrm{M}$ JAK inhibitor I for 30 min. (B) Calu-3 cells were pretreated with the indicated concentrations of S3I-201 inhibitor for 30 min. The cells were washed with PBS and then infected with SARS-CoV-2 in PBS at $0.5 \mathrm{MOI}$. After $1 \mathrm{~h}$ of incubation, the medium was replenished with DMEM containing $2 \%$ FBS. (C) Calu-3 cells were treated with 0.1\% DMSO, $10 \mu \mathrm{M}$ PD169316, $25 \mu \mathrm{M}$ PD98059, $25 \mu \mathrm{M}$ SP600125, $25 \mu \mathrm{M}$ AG490, $1 \mu \mathrm{M}$ JAK inhibitor I, or $20 \mu \mathrm{M}$ S3I-201. (A-C) After $48 \mathrm{~h}$ of incubation, cell lysates were prepared, and Western blotting was performed with the indicated antibodies (D) Calu-3 cells were pretreated with 0.1\% DMSO, $25 \mu \mathrm{M}$ AG490, $1 \mu \mathrm{M}$ JAK inhibitor I, $20 \mu \mathrm{M}$ S3I-201, or $10 \mu \mathrm{M}$ STA-21 for 30 min. The cells were washed with PBS and then infected with SARS-CoV-2 in PBS at $0.5 \mathrm{MOI}$. After $1 \mathrm{~h}$ of incubation, the medium was replenished with DMEM containing 2\% FBS. After $48 \mathrm{~h}$ of incubation, the cells were treated with $20 \mathrm{nM}$ Leptomycin B for $3 \mathrm{~h}$. To evaluate phosphorylation and localization of STAT3, cells were fixed with $4 \%$ paraformaldehyde, stained with phospho-STAT3 antibody (green), and observed by confocal microscopy. Hoechst 33258 was used for nuclei staining (blue). 
A

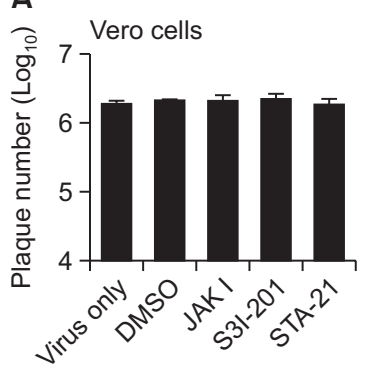

B

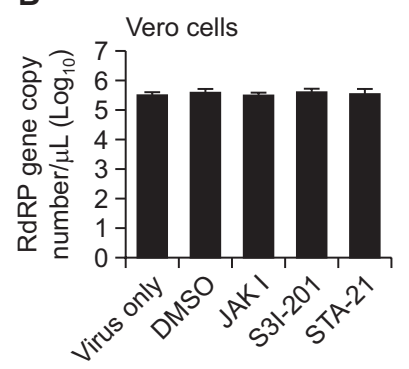

C

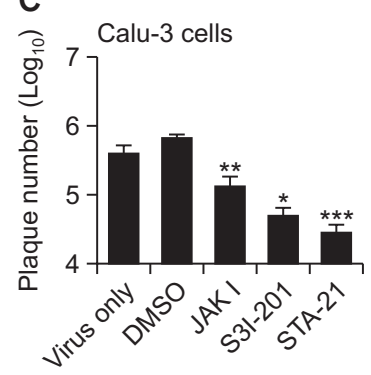

D

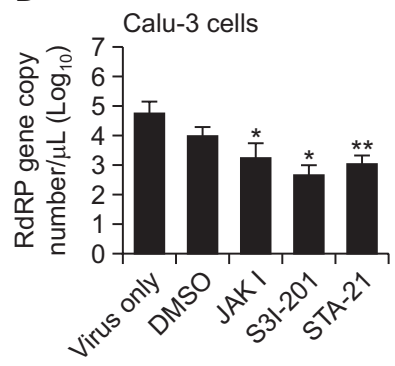

Fig. 5. Production of SARS-CoV-2 in Vero and Calu-3 cells in the presence of pathway-specific inhibitors. (A, B) Vero cells were cultured overnight in 24-well plates and pretreated with PBS, 0.1\% DMSO, $1 \mu \mathrm{M}$ JAK inhibitor I, $20 \mu \mathrm{M}$ S3I-201, or $10 \mu \mathrm{M}$ STA-21 for 30 min. The cells were washed with PBS and infected with SARS-CoV-2 in PBS $(0.1 \mathrm{MOI})$. After $1 \mathrm{~h}$ of incubation, the medium was replenished with DMEM containing 2\% FBS. After 3 days of incubation, virus production was measured by the plaque formation assay (A) and real-time RTPCR analysis of the SARS-CoV-2 RdRP gene (B). (C, D) Calu-3 cells in 12-well plates were pretreated with PBS, $0.1 \%$ DMSO, $1 \mu \mathrm{M}$ JAK inhibitor I, $20 \mu \mathrm{M} \mathrm{S3I-201}$, or $10 \mu \mathrm{M}$ STA-21 for 30 min. The cells were washed with PBS and infected with SARS-CoV-2 in PBS at 0.1 MOI. After $72 \mathrm{~h}$ of incubation, virus production was measured by the plaque formation assay (C) and real-time RT-PCR analysis of the SARSCoV-2 RdRP gene (D). ${ }^{*} p<0.05,{ }^{* *} p<0.01,{ }^{* * *} p<0.001$ compared to virus only controls.

Accordingly, virus production was higher in Vero cells than in Calu-3 cells. As higher production of virus can induce more apoptosis of cells, these results can propose different replication speed in the two cells. Although Vero cells and Calu-3 cells have ACE2, there is some difference in the mechanism of SARS-CoV-2 entry into the cells (Datta et al., 2020; Matsuyama et al., 2020). In Vero cells, the entry of SARS-CoV-2 is mediated by cathepsin B/L instead of TMPRSS2 owing to low or rare expression of TMPRSS2. In contrast, SARS-CoV-2 enters Calu- 3 cells in a TMPRSS2-dependent manner. This might affect the efficiency of virus production. However, there is another possibility that apoptosis contributes to more virus production in Vero cells because there is an active process to dephosohorylate STATs after virus infection. Apoptosis of host cells has dual functions in relation with virus infection. Protection of host cells from apoptosis is required for viruses to synthesize sufficient viral particles in the host (Kane and Golovkina, 2010). Then, apoptosis of cells at the right time is beneficial for the virus to spread viral particles to neighboring cells (Danthi, 2016). Therefore, balanced regulation of apoptosis may be one of the strategies for robust virus production. Considering the low proportion of severe cases and persistent virus propagation in some patients (Khalaf et al., 2020; Wu and McGoogan, 2020), most SARS-CoV-2 patients may release moderate levels of virus particles. Therefore, although Vero cells are useful for virus amplification, they may have limitations for use as a model system to study the pathophysiology of SARS-CoV-2. Considering that Vero cells lack competent interferon signaling because Vero cells cannot produce type I interferon, it would be a better choice to use LLC-MK2 cells, which are interferon competent African green monkey kidney cells and known to be susceptible to SARS-CoV (Kaye, 2006).

Eventually, it is necessary to study cellular responses to virus infection using primary cells in vitro and ultimately in vivo.

Because the p38 MAPK inhibitor, PD169316, and JAK inhibitor I decreased phosphorylation of STAT1 and/or STAT3 at tyrosine residues after SARS-CoV-2 infection in Calu-3 cells, p38 MAPK, JAK1, and JAK3 are likely involved in STAT activation. Therefore, unidentified SARS-CoV-2 viral proteins are likely involved in activation of p38 MAPK, JAK1, and JAK3 leading to activation of STAT1 and STAT3 in Calu-3 cells. Because MAPK phosphorylates serine or threonine residues (Biondi and Nebreda, 2003; Matsuyama et al, 2020) and because serine phosphorylation contributes to maximal STAT1 and STAT3 activity (Yokogami et al., 2000; Shuai, 2003), inhibition of p38 MAPK likely decreased STAT1 serine phosphorylation and STAT1 tyrosine phosphorylation in Calu-3 cells. Recent proteomics data showed that p38 activation and serine phosphorylation of STAT1 were induced in Vero E6 cells within $24 \mathrm{~h}$ of SARS-CoV-2 infection (McKechnie and Blish, 2020). Furthermore, pharmacological inhibitors and genetic modulations that target the p38 MAPK pathway induced antiviral activity in Vero E6 and ACE2-expressing A549 cells (Biondi and Nebreda, 2003; McKechnie and Blish, 2020). Although we investigated the effect of targeting STAT3 in this study, further studies targeting molecules in the p38 MAPK pathway and STAT1 in Calu- 3 would be informative.

The proviral functions of STAT3 have been reported for many viruses (Waris et al., 2001; Sen et al., 2012; Del Cornò et al., 2014; Roca Suarez et al., 2018), therefore STAT3 has potential as a rational target to suppress SARS-CoV-2 production. To investigate the effect of STAT3 inhibition on SARSCoV-2 production, we used pathway-specific inhibitors that target STAT3. S3I-201 and STA-21, which block dimerization and DNA binding of STAT3, reduced SARS-CoV-2 production in Calu-3 cells, but not in Vero cells. JAK inhibitor I, which suppresses phosphorylation of STAT3, also inhibited SARSCoV-2 production in Calu-3 cells, but not in Vero cells.

In summary, we investigated the regulation of STAT1 and STAT3 upon SARS-CoV-2 infection in two different cell lines and found that cellular responses to SARS-CoV-2 differ depending on the host cell type (Fig. 6). SARS-CoV-2 enters host cells, such as Vero cells and Calu- 3 cells, by binding to the cell surface receptor ACE2. SARS-CoV-2 proteins may be involved in the regulation of STAT signaling pathways. Upon SARS-CoV-2 infection of Vero cells, STAT1 and STAT3 are dephosphorylated and apoptosis occurs with high virus production. In contrast, upon SARS-CoV-2 infection of Calu-3 cells, phosphorylation of STAT1 and STAT3 persists for days after infection and proliferation-associated proteins, such as Cyclin D1 and c-Myc, are expressed with no apparent apop- 


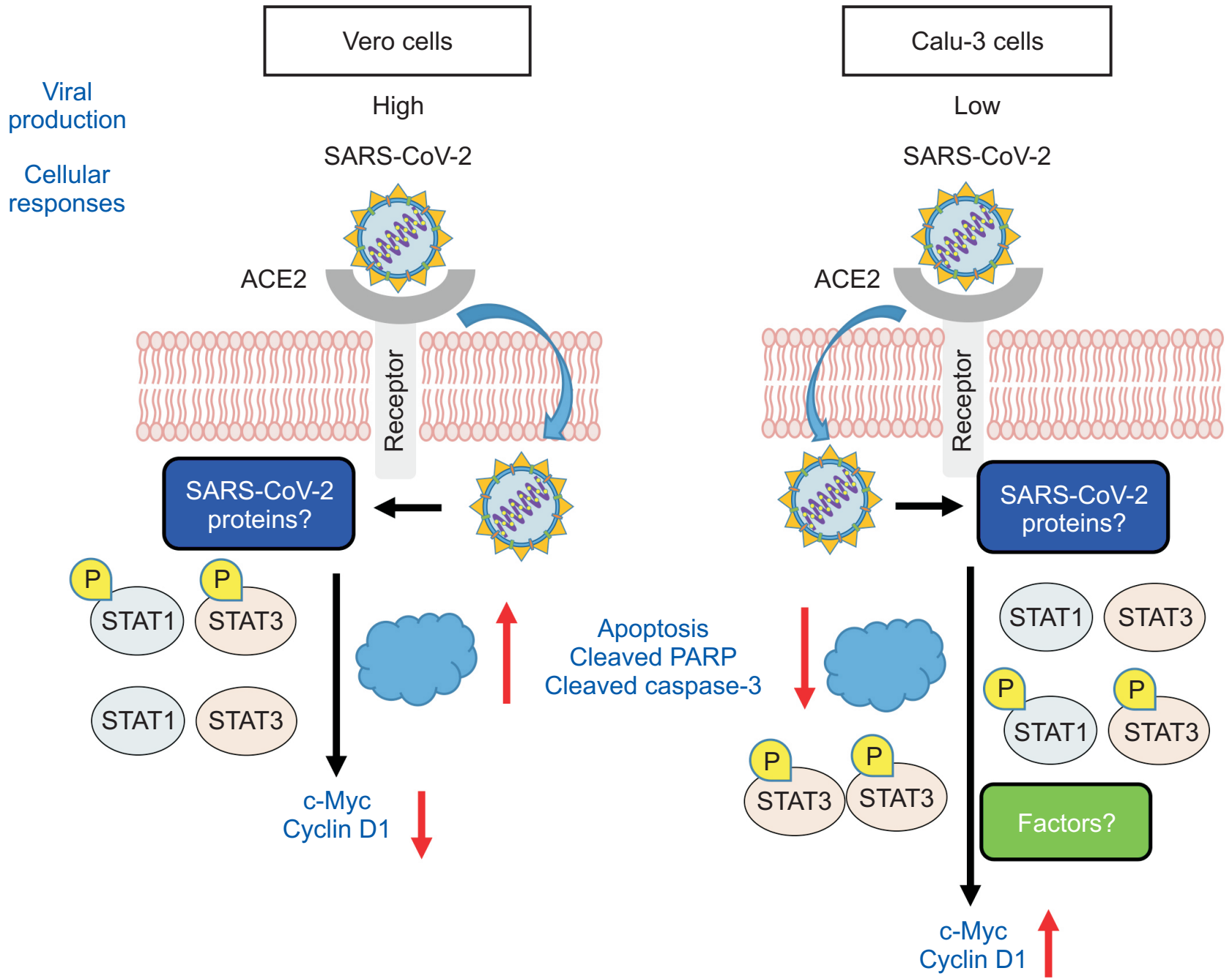

Fig. 6. Possible STAT3 signal transduction pathways in SARS-CoV-2-infected Vero Cells and Calu- 3 cells. SARS-CoV-2 enters host cells by binding to the cell surface receptor ACE2. Cellular responses to SARS-CoV-2 differ depending on the host cell type. In Vero cells, STAT1 and STAT3 are dephosphorylated within 2 days after virus infection and apoptosis occurs as observed by an increase in the amount of cleaved PARP and cleaved Caspase-3. In contrast, in Calu-3 cells, phosphorylation of STAT1 and STAT3 persists for days after virus infection and no prominent apoptosis occurs, but induction of proliferation-associated Cyclin D1 and c-Myc proteins occurs. Phosphorylation and dimerization of STAT3 are required for virus production in Calu-3 cells. Possible regulation of the STAT3 signaling pathways by SARSCoV-2 proteins is shown.

tosis and much lower virus production. In addition, phosphorylation and dimerization of STAT3 are required for virus production in Calu-3 cells. However, the inhibitors that target STAT3 had no effect on Vero cells. Although Vero cells and the derivative Vero E6 cells are widely used for SARS-CoV-2 amplification and drug screening studies, we propose a necessity to reevaluate the utility of the cells comparing with other cell lines because most appropriate cells must be used in vitro to recapitulate relevant responses to SARS-CoV-2 infection and to evaluate candidate therapeutics against SARS-CoV-2.

\section{ACKNOWLEDGMENTS}

This research was supported by grants from the $\mathrm{Na}$ tional Research Foundation (NRF-2016M3A9B6916708,
NRF-2020R1A2B5B02001806, NRF-2020M3A9I2107294) funded by the Ministry of Science and ICT in the Republic of Korea.

\section{REFERENCES}

Biondi, R. M. and Nebreda, A. R. (2003) Signalling specificity of Ser/Thr protein kinases through docking-site-mediated interactions. Biochem. J. 372, 1-13.

Chandra, V., Kar-Roy, A., Kumari, S., Mayor, S. and Jameel, S. (2008) The hepatitis $\mathrm{E}$ virus ORF3 protein modulates epidermal growth factor receptor trafficking, STAT3 translocation, and the acutephase response. J. Virol. 82, 7100-7110.

Chang, Z., Wang, Y., Zhou, X. and Long, J. E. (2018) STAT3 roles in viral infection: antiviral or proviral? Future Virol. 13, 557-574.

Danthi, P. (2016) Viruses and the diversity of cell death. Annu. Rev. 
Virol. 3, 533-553.

Datta, P. K., Liu, F., Fischer, T., Rappaport, J. and Qin, X. (2020) SARS-CoV-2 pandemic and research gaps: Understanding SARSCoV-2 interaction with the ACE2 receptor and implications for therapy. Theranostics 10, 7448-7464.

Del Cornò, M., Donninelli, G., Varano, B., Da Sacco, L., Masotti, A. and Gessani, S. (2014) HIV-1 gp120 activates the STAT3/interleukin-6 axis in primary human monocyte-derived dendritic cells. J. Virol. 88, 11045-11055.

Fehr, A. R. and Perlman, S. (2015) Coronaviruses: an overview of their replication and pathogenesis. Methods Mol. Biol. 1282, 1-23.

Girard, M. P., Cherian, T., Pervikov, Y. and Kieny, M. P. (2005) A review of vaccine research and development: human acute respiratory infections. Vaccine 23, 5708-5724.

Heim, M. H. (2015) Interferon signaling. In Signaling Pathways in Liver Diseases, 3rd ed., pp. 214-225. Wiley Blackwell, New York.

Ho, H. H. and Ivashkiv, L. B. (2006) Role of STAT3 in type I interferon responses. Negative regulation of STAT1-dependent inflammatory gene activation. J. Biol. Chem. 281, 14111-14118.

Holmes, K. V. (2003) SARS coronavirus: a new challenge for prevention and therapy. J. Clin. Invest. 111, 1605-1609.

Hui, K. P., Li, H. S., Cheung, M. C., Chan, R. W., Yuen, K. M., Mok, C. K., Nicholls, J. M., Peiris, J. S. and Chan, M. C. (2016) Highly pathogenic avian influenza $\mathrm{H} 5 \mathrm{~N} 1$ virus delays apoptotic responses via activation of STAT3. Sci. Rep. 6, 28593.

Kandeel, M., Yamamoto, M., Al-Taher, A., Watanabe, A., Oh-Hashi, K., Park, B. K., Kwon, H. J., Inoue, J. I. and Al-Nazawi, M. (2020) Small molecule inhibitors of Middle East Respiratory Syndrome Coronavirus fusion by targeting cavities on heptad repeat trimers. Biomol. Ther. (Seoul) 28, 311-319.

Kaye, M. (2006) SARS-associated coronavirus replication in cell lines. Emerg. Infect. Dis. 12, 128-133.

Kane, M. and Golovkina, T. (2010) Common threads in persistent viral infections. J. Virol. 84, 4116-4123.

Kim, J. M., Chung, Y. S., Jo, H. J., Lee, N. J., Kim, M. S., Woo, S. H., Park, S., Kim, J. W., Kim, H. M. and Han, M. G. (2020) Identification of coronavirus Isolated from a patient in Korea with COVID-19. Osong Public Health Res. Perspect. 11, 3-7.

Khalaf, K., Papp, N., Chou, J. T., Hana, D., Mackiewicz, A. and Kaczmarek, M. (2020) SARS-CoV-2: pathogenesis, and advancements in diagnostics and treatment. Front. Immunol. 11, 570927.

Kuchipudi, S. V. (2015) The complex role of STAT3 in viral infections. J. Immunol. Res. 2015, 272359.

Matsuyama, S., Nao, N., Shirato, K., Kawase, M., Saito, S., Takayama, I., Nagata, N., Sekizuka, T., Katoh, H., Kato, F., Sakata, M., Tahara, M., Kutsuna, S., Ohmagari, N., Kuroda, M., Suzuki, T., Kageyama, T. and Takeda, M. (2020) Enhanced isolation of SARS-CoV-2 by TMPRSS2-expressing cells. Proc. Natl. Acad. Sci. U.S.A. 117, 70017003.

McKechnie, J. L. and Blish, C. A. (2020) The innate immune system: fighting on the front lines or fanning the flames of COVID-19? Cell Host Microbe 27, 863-869.

Mizutani, T., Fukushi, S., Murakami, M., Hirano, T., Saijo, M., Kurane, I. and Morikawa, S. (2004) Tyrosine dephosphorylation of STAT3 in SARS coronavirus-infected Vero E6 cells. FEBS Lett. 577, 187192.

Nan, Y., Wu, C. and Zhang, Y. J. (2017) Interplay between Janus kinase/signal transducer and activator of transcription signaling activated by type I interferons and viral antagonism. Front. Immunol. 8, 1758.

Park, A. and Iwasaki, A. (2020) Type I and type III Interferons - induction, signaling, evasion, and application to combat COVID-19. Cell Host Microbe 27,870-878.
Park, B. K., Maharjan, S., Lee, S. I., Kim, J., Bae, J. Y., Park, M. S. and Kwon, H. J. (2019) Generation and characterization of a monoclonal antibody against MERS-CoV targeting the spike protein using a synthetic peptide epitope-CpG-DNA-liposome complex. BMB Rep. 52, 397-402.

Peiris, J. S., Guan, Y. and Yuen, K. Y. (2004) Severe acute respiratory syndrome. Nat. Med. 10, S88-S97.

Rawlings, J. S., Rosler, K. M. and Harrison, D. A. (2004) The JAK/ STAT signaling pathway. J. Cell Sci. 117, 1281-1283.

Roca Suarez, A. A., Van Renne, N., Baumert, T. F. and Lupberger, J. (2018) Viral manipulation of STAT3: evade, exploit, and injure. PLoS Pathog. 14, e1006839.

Rothan, H. A. and Byrareddy, S. N. (2020) The epidemiology and pathogenesis of coronavirus disease (COVID-19) outbreak. J. Autoimmun. 109, 102433.

Sen, N., Che, X., Rajamani, J., Zerboni, L., Sung, P., Ptacek, J. and Arvin, A. M. (2012) Signal transducer and activator of transcription 3 (STAT3) and survivin induction by varicella-zoster virus promote replication and skin pathogenesis. Proc. Natl. Acad. Sci. U.S.A. 109, 600-605.

Shang, J., Wan, Y., Luo, C., Ye, G., Geng, Q., Auerbach, A. and Li, F. (2020) Cell entry mechanisms of SARS-CoV-2. Proc. Natl. Acad. Sci. U.S.A. 117, 11727-11734.

Shuai, K. (2003) Serine phosphorylation: arming Stat1 against infection. Immunity 19, 771-772.

Wang, W. B., Levy, D. E. and Lee, C. K. (2011) STAT3 negatively regulates type I IFN-mediated antiviral response. J. Immunol. 187, 2578-2585.

Waris, G., Huh, K. W. and Siddiqui, A. (2001) Mitochondrially associated hepatitis $B$ virus $X$ protein constitutively activates transcription factors STAT-3 and NF-kappa B via oxidative stress. Mol. Cell. Biol. 21, 7721-7730.

WHO (2015). Summary of Probable SARS Cases with Onset of Illness from 1 November 2002 to 31 July 2003. Available from: https:// www.who.int/csr/sars/country/table2004_04_21/en/.

WHO (2019). Middle East Respiratory Syndrome Coronavirus (MERSCoV). Available from: http://www.who.int/emergencies/mers-cov/ en/.

WHO (2020). Coronavirus disease (COVID-19) Weekly Epidemiological Update and Weekly Operational Update. Available from: https:// www.who.int/emergencies/diseases/novel-coronavirus-2019/situation-reports/.

Wu, Z. and McGoogan, J. M. (2020) Characteristics of and important lessons from the Coronavirus disease 2019 (COVID-19) outbreak in china: summary of a report of 72314 cases from the Chinese center for disease control and prevention. JAMA 323, 1239-1242.

Xia, S., Liu, M., Wang, C., Xu, W., Lan, Q., Feng, S., Qi, F., Bao, L., Du, L., Liu, S., Qin, C., Sun, F., Shi, Z., Zhu, Y., Jiang, S. and Lu, L. (2020) Inhibition of SARS-CoV-2 (previously 2019-nCoV) infection by a highly potent pan-coronavirus fusion inhibitor targeting its spike protein that harbors a high capacity to mediate membrane fusion. Cell Res. 30, 343-355.

Yokogami, K., Wakisaka, S., Avruch, J. and Reeves, S. A. (2000) Serine phosphorylation and maximal activation of STAT3 during CNTF signaling is mediated by the rapamycin target mTOR. Curr. Biol. 10, 47-50.

Zaki, A. M., van Boheemen, S., Bestebroer, T. M., Osterhaus, A. D. and Fouchier, R. A. (2012) Isolation of a novel coronavirus from a man with pneumonia in Saudi Arabia. N. Engl. J. Med. 367, 1814-1820.

Zhang, E. X., Oh, O. S., See, W., Raj, P., James, L., Khan, K. and Tey, J. S. (2016) Assessment of the risk posed to Singapore by the 2015 Middle East respiratory syndrome outbreak in the Republic of Korea. Western Pac. Surveill. Response J. 7, 17-25. 